\title{
Presencia del Virus Papiloma Humano en la Cavidad Oral: Revisión y Actualización de la Literatura
}

\author{
Presence of Human Papillomavirus in Oral Cavity: Review and Update of Literature \\ Perla Cháirez Atienzo*; María Elisa Vega Memíje*; Graciela Zambrano Galván**; \\ Alma Graciela García Calderón ${ }^{\star * * *}$; Ixchel Araceli Maya García ${ }^{\star \star * * *}$ \& Juan Carlos Cuevas González
}

CHÁIREZ, A. P.; VEGA, M. M. E.; ZAMBRANO, G. G.; GARCíA, C. A. G.; MAYA, G. I. A \& CUEVAS, G. J. C. Presencia del virus papiloma humano en la cavidad oral: Revisión y actualización de la literatura. Int. J. Odontostomat., 9(2):233-238, 2015.

RESUMEN: El virus del papiloma humano (VPH) forma parte de un grupo de virus ADN heterogéneo llamados papillomaviridae; este virus es causante de múltiples lesiones hiperplásicas, verrucosas y papilomatosas de las células epiteliales de piel y mucosas, existen más de 120 tipos de VPH, de solo 100 se conoce su secuencia genómica completa. Los dos géneros más importantes son los papilomavirus alpha ( $\partial$ ) y los beta (ß), la mayoría de los virus que infectan el área genital pertenecen al género alpha; según sus características clínicas, se pueden subdividir en cutáneos y de mucosa. Si bien las infecciones por este virus son frecuentes en cavidad oral, este campo de la medicina se encuentra en estudio, debido a que la información sobre el tema no es concluyente, es prioritario para el estomatólogo conocer las generalidades acerca del VPH y tratar las lesiones de una manera informada, este virus se asocia a un 35\% de los casos de cáncer de cavidad oral, de los cuales el $70 \%$ de ellos son de alto riesgo. En México, se tiene estimado que cerca del $43 \%$ de los hombres y del $17,5 \%$ de las mujeres, todos ellos sanos y sexualmente activos tienen alguna infección por VPH.

PALABRAS CLAVE: virus papiloma humano; cavidad oral; lesiones cutáneas y/o de mucosas.

\section{INTRODUCCIÓN}

El virus del papiloma humano (VPH) forma parte de un grupo de virus ADN heterogéneo llamados papillomaviridae; este virus, es causante de múltiples lesiones hiperplásicas, verrucosas y papilomatosas de las células epiteliales de piel y mucosas. Tiene un tamaño aproximado de $50 \mathrm{~nm}$ de diámetro. Su única molécula de $A D N$ de doble cadena, presenta aproximadamente 8,000 pares de bases. Existen más de 120 tipos de VPH, algunos tipos se muestran en la Tablal, de solo 100 se conoce su secuencia genómica completa (García-Cuellar et al., 2004). Los dos géneros de VPH más importantes son los papilomavirus alpha $(\partial)$ y los beta $(ß)$, la mayoría de los virus que infectan el área genital pertenecen al género alpha (Lizano et al., 2009), según sus características clínicas, los VPH se pueden subdividir en cutáneos y de mucosa (Castillo, 2011).

\begin{tabular}{ccl} 
Alto Riesgo & Bajo Riesgo & \\
\cline { 1 - 2 } 16 & 6 & \\
18 & 11 & \\
26 & 40 & \\
31 & 42 & \\
33 & 43 & \\
35 & 44 & \\
39 & 54 & \\
45 & 61 & \\
51 & 72 & \\
52 & 81 & \\
53 & CP6108 & \\
56 & & Tabla I. Distintos tipos \\
58 & & de VPH agrupados \\
59 & & según el riesgo que \\
66 & & representan (Muñoz \\
68 & & et al., 2003). \\
82 & &
\end{tabular}

Alumna de la Maestría en Ciencias Estomatológicas, Universidad Juárez del Estado de Durango, Durango, México.

* Dermatopatóloga, Subdirectora de Investigación, Hospital General Dr. Manuel Gea González, México DF, México.

*** Profesor Investigador, Facultad de Odontología, Universidad Juárez del Estado de Durango, Durango, México.

**** Cirujano Dentista Especialista, Práctica Privada, México DF, México.

**o*** Profesora Investigadora, Facultad de Odontología, Universidad Autónoma de Campeche, Campeche, México. 
CHÁIREZ, A. P.; VEGA, M. M. E.; ZAMBRANO, G. G.; GARCÍA, C. A. G.; MAYA, G. I. A \& CUEVAS, G. J. C. Presencia del virus papiloma humano en la cavidad oral: Revisión y actualización de la literatura. Int. J. Odontostomat., 9(2):233-238, 2015.

Tabla II. VPH encontrados en lesiones de cavidad oral.

\begin{tabular}{|c|c|c|c|c|c|c|c|c|c|c|c|c|c|c|c|}
\hline \multicolumn{16}{|c|}{ Tipos de VPH } \\
\hline 1 & 2 & 3 & 4 & 5 & 6 & 7 & 10 & 13 & 16 & 18 & 31 & 32 & 33 & 35 & 57 \\
\hline
\end{tabular}

Tabla III. Lesiones en donde se ha encontrado la presencia de algunos tipos de VPH.

\begin{tabular}{cc}
\hline Lesiones benignas & $\begin{array}{c}\text { Lesiones potencialmente } \\
\text { cancerizables y/o malignas }\end{array}$ \\
\hline $\begin{array}{c}\text { Papiloma bucal } \\
\text { Verruga vulgar }\end{array}$ & $\begin{array}{c}\text { Leucoplasia idiopática } \\
\text { Condiloma acuminado }\end{array}$ \\
Hiperplasia epitelial multifocal & \\
\hline
\end{tabular}

Como se muestra en la Tabla II, en lesiones de la cavidad oral se han encontrado 16 tipos de VPH siendo exclusivos de la cavidad oral los subtipos 13 y 32 (Serena et al., 2011).

El VPH es un factor de riesgo para desarrollar cáncer cervicouterino y algunas verrugas en piel y mucosas. Se han llevado a cabo numerosas investigaciones en esta área, lo cual nos lleva a tener amplio acceso a esta información; sin embargo, no pasa lo mismo con la estomatología, aunque las infecciones por este virus son frecuentes en la cavidad oral, este campo de la medicina se encuentra en estudio, debido a esto la información sobre el tema no es concluyente, esta situación nos ha llevado a realizar una revisión y actualización de la literatura referente a la presencia del VPH en la cavidad oral, con la finalidad de que el estomatólogo conozca las generalidades acerca del VPH y trate las lesiones de una manera informada.

Estudios previos muestran que el VPH se asocia a un $35 \%$ de los casos de cáncer de cavidad oral, de los cuales el $70 \%$ de ellos son de alto riesgo (Gallegos et al., 2007). Se estima que las infecciones son las causantes de un $17,8 \%$ de casos de cáncer en el mundo; de un $26,3 \%$ de lesiones malignas en países económicamente desarrollados y del $7,7 \%$ en países en vías de desarrollo (Castillo).

El VPH es una de las infecciones de transmisión sexual con más prevalencia en la actualidad; en México, se tiene estimado que cerca del $43 \%$ de los hombres y del $17,5 \%$ de las mujeres, todos ellos sanos y sexualmente activos tienen alguna infección por VPH (Ibieta et al., 2005). Según algunos estudios, las personas pueden adquirir el VPH en el período perinatal por infección transplacentaria, por líquido amniótico, durante el contacto sexual, o por auto-ino- culación. Una posible transmisión del virus por medio de la saliva ha sido sugerida también por algunos autores (Bologna-Molina et al., 2006).

Se sugiere que las infecciones orales por algunos tipos de VPH se transmiten durante el acto sexual oral, por lo que la conducta sexual, el número de parejas sexuales y la edad temprana de inicio de la actividad sexual son factores determinantes para contraer dicha infección; y se ha demostrado que el virus que infecta el área genital puede también infectar la cavidad oral (Sánchez-Vargas et al., 2010).

Las lesiones producidas por estos virus se pueden clasificar en dos grandes grupos (ver Tabla III), lesiones benignas y lesiones potencialmente cancerizables o malignas (Luciano \& Oviedo, 2013). Las cuales se describen a continuación:

Papiloma oral. Lesión benigna que afecta a niños y adultos, pero tiene cierta predilección por personas de 30 a 50 años, los sitios de localización son: lengua, paladar blando, úvula, frenillo y bermellón (Fig. 1). Clínicamente presentan un aspecto parecido a la coliflor, una superficie digitiforme, con crecimiento exofítico y base sésil. Su coloración depende del grado de queratinización y puede ir del blanco al rosado. Por lo general son lesiones solitarias, indoloras, de un tamaño aproximado de $1 \mathrm{~cm}$ de diámetro y de crecimiento rápido, una forma de contagio del VPH es por contacto directo, estas lesiones son ocasionadas principalmente por los subtipos de VPH 6 y 11 (Neville \& Bussoloti Filho, 2002; Castro \& Bussoloti Filho, 2006; Jiménez \& Pérez, 2002).

Verruga vulgar. Es una lesión benigna, papilomatosa escamosa, que se localiza frecuentemente en lengua, paladar duro, paladar blando, labio y bermellón, común en niños y adolescentes; su prevalencia es igual en 


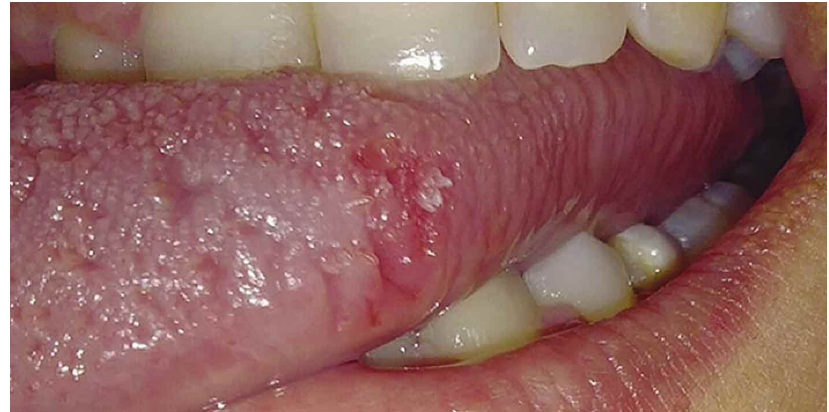

Fig. 1. Papiloma oral, lesión exofítica de base sésil en margen lateral de lengua (VPH +11).

hombres y mujeres. Se presenta como lesión asintomática, de consistencia firme, crecen rápidamente hasta alcanzar un tamaño máximo de 5 a $6 \mathrm{~mm}$, su coloración va del blanco al rosa dependiendo del grado de queratinización, son lesiones generalmente solitarias pero pueden presentarse de manera múltiple. Se ha sugerido que estas lesiones se contagian por contacto directo entre las personas, pero también pueden surgir mediante la autoinoculación, ya que por lo general se presentan en personas con verrugas en manos y dedos. Clínicamente son lesiones muy parecidas al papiloma oral, por lo que su diagnóstico de verruga oral debe de corresponder al de una verruga cutánea ordinaria, pero se debe confirmar la presencia de algún tipo de VPH (Flores Ramos \& Strauss Quintela, 2010).

La verruga vulgar se encuentra asociada preferentemente con los subtipos del virus del papiloma humano 2, 57 (Castro \& Bussoloti Filho), 4, 6, 40 (Neville et al.). Para el tratamiento de verrugas cutáneas y ano-genitales se ha propuesto el 5-fluoracilo, crioterapia, electro-cirugía, extirpación quirúrgica y tratamiento con láser, en los casos en los que se opta por electro-cirugía y cirugía con láser se deben de utilizar mascarillas quirúrgicas y extractores de humo dado la presencia de virus viable en los extractores (Concha, 2007).

Condiloma acuminado. Son lesiones que se pueden presentar en la mucosa oral, su apariencia es nodular blanda y sésil, con una superficie parecida a la coliflor, su tamaño puede variar (Fig. 2), su forma de contagio es venérea o por auto-inoculación. Histológicamente son lesiones de epitelio escamoso estratificado con acantosis y escasa paraqueratosis, con prolongaciones epiteliales densas y elongadas, es frecuente observar células coilociticas en la capa córnea y espinosa del epitelio (Luciano \& Oviedo). Los subtipos de VPH que causan el condiloma acuminado son el 6, 11 y 42 (Pérez-Salcedo \& Bascones Martinez, 2010). El tratamiento es la escisión quirúrgica que puede efectuarse mediante criocirugía, escisión con bisturí, electrodesecación o ablación con láser, las recurrencias son comunes relacionadas con el tejido circundante de aspecto normal que puede alojar al agente infeccioso (Regezi \& Sciubba 1991).

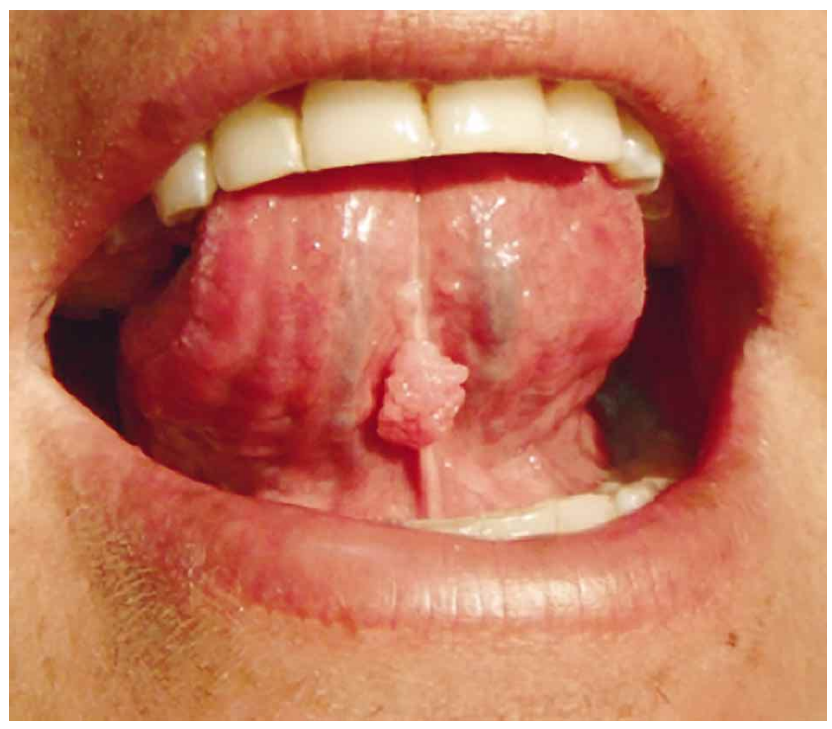

Fig. 2. Condiloma en superficie ventral de la lengua en paciente de sexo femenino (VPH +42).

Hiperplasia epitelial multifocal. También conocida como enfermedad de Heck. Como se muestra en la Figura 3 es una patología benigna de la mucosa oral, caracterizada por lesiones múltiples, bien definidas, de un tamaño aproximado de $5 \mathrm{~mm}$, estas lesiones se presentan a manera de pápulas o placas del mismo color de la mucosa oral. Afecta labios, margen lateral de la lengua, así como la mucosa, su crecimiento es lento, y principalmente se presentan en niños de ambos sexos (González-Losa et al., 2011).

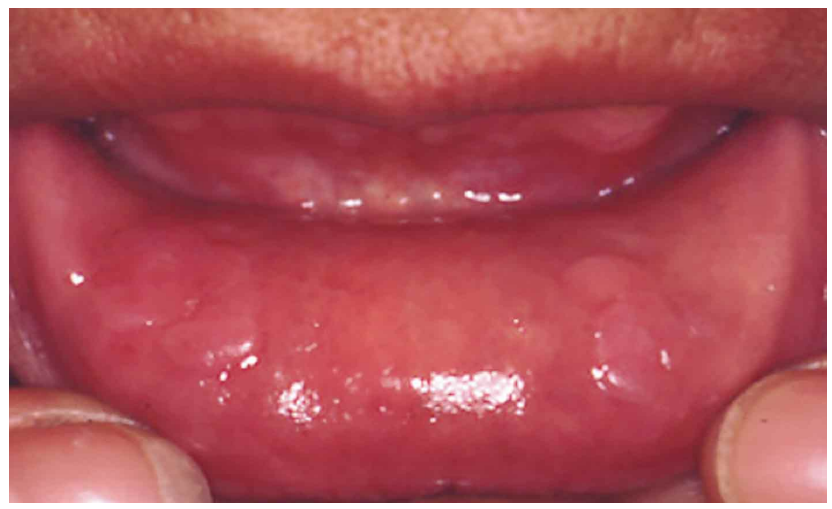

Fig. 3. Lesiones de hiperplasia epitelial multifocal, elevadas y bien delimitadas localizadas en la mucosa del labio inferior en paciente que acudió a revisión médica. 
Estas lesiones se han reportado sobretodo en grupos étnicos latinoamericanos, se han asociado a múltiples factores tales como predisposición genética, desnutrición, higiene y las condiciones de vivienda de las personas afectadas (Carlos \& Sedano, 1994). Algunos estudios han revelado la presencia del VPH en estas lesiones, el primero en reportarse fue el subtipo 13 y es el que se encuentra con más frecuencia; pero también, se ha descrito la presencia del subtipo 32 (García-Corona et al., 2004; González-Losa et al.), aunque algunos estudios demostraron la presencia de los subtipos 11 y 16 (Ledesma-Montes et al., 2005). Si bien las lesiones permanecen autolimitadas a largo plazo se ha propuesto la extirpación quirúrgica sobre todo si interfieren con la masticación, la suplementación con vitamina E, criocirugía e Imiquimod al 5\% (de la Teja-Ángeles et al., 2008).

Con respecto a las lesiones potencialmente cancerizables tenemos a la leucoplasia oral idiopática, la cual se divide por su apariencia clínica en homogénea y no homogénea; el alcohol y el tabaco, son los factores de riesgo más comúnmente asociados con esta lesión.

Una vez que la leucoplasia se presenta y el diagnóstico histopatológico es de displasia leve o moderada puede tener una regresión espontánea, o progresar a carcinoma, desconociéndose las causas de esto. Estudios epidemiológicos estiman que la prevalencia mundial de leucoplasia es de $0,5 \%$ a 3,46\%, y establecen que una leucoplasia se transforma en carcinoma en un $0,7 \%$ a $2,9 \%$ de las veces aunque los datos son distintos en la literatura. Se caracteriza por ser una lesión blanca, firme o queratósica no removible en la mucosa oral, su tamaño varía de varios milímetros hasta algunos centímetros de diámetro, las zonas más afectadas por esta lesión en la cavidad oral son paladar duro y blando, lengua y piso (Feller \& Lemmer, 2012). Los subtipos de VPH asociados a la leucoplasia son los 16 y 18 (Marsán Suárez et al., 2010).

El carcinoma oral y orofaríngeo son los tipos más comunes de cáncer de cabeza y cuello, y más del $90 \%$ de éstos son carcinomas de células escamosas; ha sido asociado a factores de riesgo como el tabaco y el alcohol entre otros (Ibieta-Zarco et al., 2012). Los sitios afectados con mayor frecuencia son la lengua, seguido por la encía y piso de cavidad oral (Anaya-Saavedra et al., 2008).

Estudios epidemiológicos indican que algunos subtipos del VPH están presentes en esta neoplasia, cabe mencionar que el VPH hasta el momento no es considerado un factor de riesgo para desarrollar COCE, pero se requieren estudios que diluciden el papel del virus en la etiología de esta neoplasia.

El carcinoma de cabeza y cuello, afecta por lo general a pacientes de edad avanzada, particularmente aquellos que cuentan con un historial amplio de ingesta de alcohol y uso de tabaco; sin embargo actualmente afecta también a personas cada vez más jóvenes e incluso sin factores de riesgo asociados, por lo que se ha propuesto que el estado inmunológico y hormonal del paciente debe de ser tomado en cuenta así como el comportamiento sexual, número de parejas sexuales y el papel que desempeñan las modificaciones epigenéticas en el desarrollo del cáncer oral (González-Ramírez et al., 2013).

La International Agency for Research on Cancer (IARC) estableció la presencia de VPH en 3,9\% de los cánceres orales y el $18,3 \%$ en los carcinomas de células escamosas en orofaringe (Ibieta et al.). Los subtipos de VPH 16 y 18 se encuentran en un $80 \%$ de los cánceres, mientras que los subtipos 31,33 y 35 son menos frecuentes encontrándose sólo en un 3\% (BolognaMolina et al).

Histopatológicamente las lesiones papilomatosas se caracterizan por la presencia de una proliferación de epitelio escamoso estratificado queratinizado, donde se encuentran proyecciones digitiformes con centros fibrovasculares de tejido conectivo, el centro del tejido conectivo puede mostrar cambios inflamatorios, dependiendo del trauma sufrido por la lesión. Las lesiones con apariencia clínica blanquecina presentan capas de queratina más gruesa, se puede encontrar en la capa de células espinosas un alto concentrado de coilocitos, que son células epiteliales con núcleos pequeños, oscuros y halo claro característico de VPH. En algunos papilomas se puede encontrar hiperplasia basilar, así como actividad mitótica, que puede ser confundida con una displasia epitelial leve (Neville et al).

El diagnóstico de las lesiones papilomatosas se enfoca en el examen clínico, para determinar su localización anatómica así como las características histopatológicas. Otro auxiliar en el diagnóstico de estas lesiones son las técnicas de biología molecular, que se utilizan para la tipificación de VPH, estas técnicas incluyen Hibridación in situ (HIS), captura de híbridos $(\mathrm{HC})$ y la Amplificación génica por medio de la reacción en cadena de la polimerasa (PCR) (Jaled \& Moreno. 2010). 


\section{CONCLUSIONES}

Debido a la escasa información disponible sobre la presencia del VPH en la cavidad oral es imperativa la elaboración de estudios científicos sobre el tema y que el estomatólogo esté familiarizado con este tipo de lesiones para que las pueda tratar de una manera informada.

CHÁIREZ, A. P.; VEGA, M. M. E.; ZAMBRANO, G. G.; GARCÍA, C. A. G.; MAYA, G. I. A \& CUEVAS, G. J. C. Presence of human papillomavirus in oral cavity: Review and update of literature. Int. J. Odontostomat., 9(2):233238, 2015.

ABSTRACT: The human papillomavirus (HPV) is part of heterogeneous group of DNA viruses called papillomaviridae; this virus causes multiple hyperplastic, papillomatous and warty lesions of epithelial cells of skin and mucosa, there are more than 120 types of HPV, although, the complete genome sequence of only 100 of these is known. The most important types are the alpha papillomavirus (a) and beta (b), most viruses that infect the genital area belong to the alpha type; according to their clinical characteristics, they can be divided in cutaneous and mucosal. HPV infections are common in oral cavity, this field of medicine is currently being studied, considering that the information about this subject is not conclusive, it is a priority for the stomatologist to know the generalities about HPV and treat injuries in an informed manner; this virus is associated to a $35 \%$ of cases of oral cancer, of which $70 \%$ are considered high risk. In Mexico, it is estimated that about a $43 \%$ of men and a $17.5 \%$ of women, all of them healthy and sexually active have at least one HPV infection.

KEY WORDS: human papillomavirus; oral cavity, skin lesions.

\section{REFERENCIAS BIBLIOGRÁFICAS}

Anaya-Saavedra, G.; Ramírez-Amador, V.; IrigoyenCamacho, M. E.; García-Cuellar, C. M.; Guido-Jiménez, M.; Méndez-Martínez, R. \& García-Carrancá, A. High association of human papillomavirus infection with oral cancer: a case-control study. Arch. Med. Res., 39(2):18997, 2008.

Bologna-Molina, R. E.; Castañeda-Castaneira, R. E.; MolinaFrechero, N. \& Pérez Frechero, E. Virus del Papiloma humano y su asociación con el cáncer bucal. Rev. Med. Inst. Mex. Seguro Soc., 44(2):147-53, 2006.
Carlos, R. \& Sedano, H. O. Multifocal papilloma virus epithelial hyperplasia. Oral Surg. Oral Med. Oral Pathol., 77(6):631-5, 1994.

Castillo, A. HPV infection and carcinogenesis in the upper aero-digestive tract. Colomb. Med., 42(2):233-42, 2011.

Castro, T. P. \& Bussoloti Filho, I. Prevalence of human papillomavirus (HPV) in oral cavity and oropharynx. Braz. J. Otorhinolaryngol., 72(2):272-82, 2006.

Concha, R. M. Diagnóstico y terapia del virus papiloma humano. Rev. Chil. Infect., 24(3):209-14, 2007.

de la Teja-Ángeles, E.; Martínez-Sandoval, B.; TéllezRodríguez, J.; Ramírez-Paredes, L. F.; Durán-Gutiérrez, A. \& Cadena-Galdós, A. Hiperplasia epitelial multifocal. Manifestaciones bucales en niños. Revisión de la literatura. Acta Pediatr. Mex., 29(1):31-5, 2008.

Feller, L. \& Lemmer, J. Oral Leukoplakia as It Relates to HPV Infection: A Review. Int. J. Dent., 2012:540561, 2012.

Flores Ramos, J. L. \& Strauss Quintela, M. Papilomas orales: un análisis clínico en pacientes que acuden a la clínica Odontológica Univalle-La Paz. Rev. Inv. Info Salud, 5(12):15-24, 2010.

Gallegos, H. J.; Minauro, M. G.; Apresa, T.; Arias, C. H.; Hernández, S. M.; Flores, D. R.; Reséndiz, C. J. \& Hernández, H. D. M. Cáncer de lengua y virus del papiloma humano (VPH). Acta Méd. Grupo Ángel., 5(3):129-33, 2007

García-Corona, C.; Vega-Memije, E.; Mosqueda-Taylor, A.; Yamamoto-Furusho, J. K.; Rodríguez-Carreón, A. A.; Ruiz-Morales, J. A.; Salgado, N. \& Granados, J. Association of HLA-DR4 (DRB $\left.1^{*} 0404\right)$ with human papillomavirus infection in patients with focal epithelial hyperplasia. Arch. Dermatol., 140(10):1227-31, 2004.

García-Cuellar, C. M.; González Ramírez, I. C. \& Granados García, M. VPH y los carcinomas de cavidad bucal y bucofaringe. Cancerol., 4:181-91, 2004.

González-Ramírez, I.; Irigoyen-Camacho, M. E.; RamírezAmador, V.; Lizano-Soberón, M.; Carrillo-García, A.; García-Carrancá, A.; Sánchez-Pérez, Y.; MéndezMartínez, R.; Granados-García, M.; Ruíz-Godoy, L. \& García-Cuellar, C. Association between age and highrisk human papilloma virus in Mexican oral cancer patients. Oral Dis., 19(8):796-804, 2013.

González-Losa, M. R.; Suarez-Allén, R. E.; Canul-Canche, J.; Conde-Ferráez, L. \& Eljure-Lopez, N. Multifocal epithelial hyperplasia in a community in the Mayan area of Mexico. Int. J. Dermatol., 50(3):304-9, 2011. 
Ibieta, B. R.; Lizano, M.; Fras-Mendivil, M.; Barrera, J. L.; Carrillo, A.; Ma Ruz-Godoy, L. \& Mohar, A. Human papilloma virus in oral squamous cell carcinoma in a Mexican population. Oral Surg. Oral Med. Oral Pathol. Oral Radiol. Endod., 99(3):311-5, 2005.

Ibieta-Zarco, B. R.; Carrillo-García, A.; Ponce-de-León-Rosales, S.; Flores-Miranda, M. M.; Mohar, A. \& Lizano, M. Frequency and genotype distribution of multiple human papillomavirus infections in cancer of the head and neck in a Mexican population. Oral Surg. Oral Med. Oral Pathol. Oral Radiol., 114(3):350-7, 2012.

Jaled, M. M. \& Moreno, H. C. Virus Papiloma Humano (HPV). Parte II - Clínica y terapéutica. Dermatol. Argent., 16(2):102-8, 2010.

Jiménez, C. \& Pérez, C. Estudio clínico-patológico retrospectivo de papiloma de la mucosa bucal en una población venezolana. Acta Odontol. Venez., 40(1):31-5, 2002.

Ledesma-Montes, C.; Vega-Memije, E.; Garcés-Ortíz, M.; Cardiel-Nieves, M. \& Juárez-Luna, C. Multifocal epithelial hyperplasia. Report of nine cases. Med. Oral Patol. Oral Cir. Bucal, 10(5):394401, 2005.

Lizano-Soberón, M.; Carrillo-García, A. \& ContrerasParedes, A. Infección por virus del papiloma humano: epidemiología, historia natural y carcinogénesis. Cancerol., 4:205-16, 2009.

Luciano, R. \& Oviedo, J. M. Virus del Papiloma Humano y cáncer bucal. Acta Odontol. Venez., 51(1):1-3, 2013.

Marsán Suárez, V.; Valcárcel Llerandi, J.; Macías Abraham, C.; Sánchez Segura, M.; Insua Arregui, C.; del Valle Pérez, L.; Beatriz Socarrás Ferrer, B.; Palma Salgado, L.; Arce Hernández, A. \& García Cuéllar, M. Leucoplasia oral en un paciente pediátrico con deficiencia primaria de linfocitos $T$. Rev. Cuba. Hematol. Inmunol. Hemoter., 26(1):629, 2010.

Muñoz, N.; Bosch, F. X.; de Sanjosé, S.; Herrero, R.; Castellsagué, X.; Shah, K. V.; Snijders, P. J.; Meijer, C. J. \& International Agency for Research on Cancer Multicenter Cervical Cancer Study Group. Epidemiologic classification of human papillomavirus types associated with cervical cancer. N. Engl. J. Med., 348(6):518-27, 2003.
Neville, B. W.; Damm, D. D.; Allen, C. M. \& Bouquot, J. E. Oral and Maxillofacial Pathology. 2nd ed. Philadelphia, W. B. Saunders, 2002.

Pérez-Salcedo, L. \& Bascones Martinez, A. Tumores Benignos de la mucosa oral. Av. Odontoestomatol., 26(1):11-8, 2010.

Regezi, J. A.; Sciubba, J. J. \& Jordan, R. C. Oral Pathology: Clinical Pathologic Correlations. 4th Ed. Philadelphia, W. B. Saunders, 2003.

Sánchez-Vargas, L. O.; Díaz-Hernández, C. \& Martinez-Martinez, A. Detection of Human Papilloma Virus (HPV) in oral mucosa of women with cervical lesions and their relation to oral sex practices. Infect. Agent. Cancer, 5(1):25, 2010.

Serena-Gómez, E.; Bologna-Molina Ronell, E.; Nevarez-Rascon, A. \& Rocha Buelvas, A. HPV Prevalence on Malignant Lesion Process of the Upper Aerodigestive Tract. Int. J. Odontostomat., 5(1):5-12, 2011.

Dirección para Correspondencia:

Dr. Juan Carlos Cuevas González

Posgrado e investigación, Facultad de Odontología

Universidad Juárez del Estado de Durango

AV. Predio Canoas S/N Colonia los Ángeles

C.P 34000, Durango,

MÉXICO

Email: cuevas_gonzalez@hotmail.com

Recibido :08-04-2015

Aceptado:14-07-2015 